CCNY-HEP-04/8

August 2004

\title{
Chiral actions from phase space (quantum Hall) droplets
}

\author{
Alexios P. Polychronakos \\ Physics Department, City College of the CUNY \\ Convent Avenue and $138^{\text {th }}$ Street, New York, NY 10031 \\ alexios@sci.ccny.cuny.edu
}

\begin{abstract}
We derive the hamiltonian and canonical structure for arbitrary deformations of a phase space (quantum Hall) droplet on a general manifold of any dimension. The derivation is based on a transformation that decouples the Casimirs of the density Poisson structure. The linearized theory reproduces the edge state chiral action of the droplets, while the nonlinear hamiltonian captures $1 / N$ quantum corrections.
\end{abstract}




\section{Introduction}

Collective states of fermions have nontrivial dynamics and are at the source of many interesting physical phenomena. Effective descriptions of such states in terms of bosonic fields that capture their essential properties are, therefore, very desirable. Such descriptions are mostly available in one spatial dimension and particular reductions of higher dimensional systems [1]-[8].

Although fermion interactions are in general an important ingredient, there are situations in which the fermions are effectively noninteracting, the nontrivial behavior of their collective state arising from the structure of their phase space and Fermi exclusion. In such situations a fluid dynamical description of the system in terms of its phase space density becomes particularly well-suited [8]. The typical example of this is the integer quantum Hall effect, where incompressibility and edge excitations can be studied this way 9]-[13. (Fractional quantum Hall states are also amenable to a similar description but with generalized phase space statistics.)

Generalizations of the quantum Hall system to higher dimensions have been proposed by Zhang and $\mathrm{Hu}$ and led to some interesting speculations [14-[20]. This construction has been extended to various geometries, topologies and background gauge fields, and the properties of these theories have been extensively studied. In particular, Karabali and Nair gave a general method for deriving an effective action for the edge excitations of quantum Hall droplets [17]. This action is effectively chiral and, in the nonabelian case, constitutes an interesting higher dimensional generalization of the chiral sigma model known as the Wess-Zumino-Witten model [5].

The derivation of this effective action was based on the quantum mechanical density matrix formulation and a large- $N$ approximation (small perturbation of the droplet) 21. The dynamics of the quantum Hall droplet, on the other hand, are captured in an essential way by the classical fluid motion in the phase space of the droplet. The purpose of the present paper is to present a derivation of the chiral droplet action in a general setting, without the assumption of small perturbation, by analyzing their classical phase space. A nonlinear theory is obtained that describes arbitrary deformations of the droplet. Quantum mechanically, the nonlinear terms capture higher order corrections in the $1 / N$ approximation. The analysis is presented for the abelian case (spinless, colorless fermions), deferring the treatment of nonabelian droplets to a future publication.

The organization of the paper is as follows. In section 2 we give a general analysis 
of phase space density dynamics and introduce the cartographic transformation, which renders the canonical structure of the density theory amenable to a lagrangian realization. In section 3 we analyze the motion of droplets and apply the formalism of section 2 to derive their hamiltonian and Poisson structure. A linearized analysis is performed, recovering the chiral action of Karabali and Nair. In section 4 we examine the quantization of this theory in 2 dimensions and demonstrate that the expected results are obtained including corrections of order $1 / N$. Finally, section 5 contains our conclusions and discussion of outstanding issues.

\section{Phase space density dynamics}

\subsection{General formulation}

We shall consider noninteracting particles moving in a general $D$-dimensional phase space with an arbitrary hamiltonian ( $D$ must be even for a non-degenerate canonical structure). Although non-relativistic particles will be relevant for quantum Hall considerations, in principle the motion could be relativistic, depending on the structure and symmetries of the hamiltonian and phase space. External electric and magnetic fields are also included and encoded in the canonical structure.

The single-particle phase space will be described by phase space coordinates $\phi^{\alpha}$, $\alpha=0,1, \ldots D-1$ and determined by its Poisson structure:

$$
\left\{\phi^{\alpha}, \phi^{\beta}\right\}_{s p}=\theta^{\alpha \beta}
$$

where, to avoid later confusion, we put the subscript $s p$ standing for single-particle.

The Poisson matrix $\theta^{\alpha \beta}$ will be, in general, a function of the phase space coordinates $\phi^{\alpha}$. The volume element in this phase space is, then,

$$
d v=\frac{d \phi}{\sqrt{\theta}}, \text { where } \theta=\operatorname{det} \theta^{\alpha \beta}, \quad d \phi=\prod_{\alpha=0}^{D-1} d \phi^{\alpha}
$$

The single-particle hamiltonian, denoted $V(\phi)$, leads to classical motion:

$$
\dot{\phi}^{\alpha}=\left\{\phi^{\alpha}, V\right\}_{s p}=\theta^{\alpha \beta} \partial_{\beta} V
$$

A dense collection of particles on this phase space can be described in terms of its density $\rho(\phi, t)$. Under time evolution, $\rho$ changes by a canonical transformation generated by $V$ :

$$
\dot{\rho}=\{\rho, V\}_{s p}=\theta^{\alpha \beta} \partial_{\alpha} \rho \partial_{\beta} V
$$


The above equation can arise out of a hamiltonian and canonical structure for the field $\rho$. Choosing for the hamiltonian the total particle energy

$$
H=\int \frac{d \phi}{\sqrt{\theta}} \rho V
$$

the appropriate Poisson brackets are

$$
\left\{\rho\left(\phi_{1}\right), \rho\left(\phi_{2}\right)\right\}=\sqrt{\theta\left(\phi_{+}\right)} \theta^{\alpha \beta}\left(\phi_{+}\right) \partial_{\alpha} \rho\left(\phi_{+}\right) \partial_{\beta} \delta\left(\phi_{-}\right)
$$

where we defined relative and mid-point coordinates $\phi_{-}=\phi_{1}-\phi_{2}$ and $\phi_{+}=\frac{\phi_{1}+\phi_{2}}{2}$. [These brackets should not be confused with the single-particle brackets (10).] It can be checked that the canonical equation of motion for the field $\rho$ :

$$
\dot{\rho}=\{\rho, H\}
$$

reproduces equation (41). In the derivation we need to use the identity

$$
\partial_{\alpha}\left(\frac{\theta^{\alpha \beta}}{\sqrt{\theta}}\right)=0
$$

which is a corollary of the Jacobi identity for $\theta^{\alpha \beta}$.

The above brackets (6) are the standard infinite-dimensional Poisson algebra on the phase space manifold. In terms of test functions their form becomes more obvious: defining

$$
\rho[F]=\int \frac{d \phi}{\sqrt{\theta}} F(\phi) \rho(\phi)
$$

for some function on the phase space $F$, then the brackets of two such integrals are

$$
\{\rho[F], \rho[G]\}=\rho\left[\{F, G\}_{s p}\right]
$$

A lagrangian realization of the equation of motion for the field $\rho$ in terms of an action would require the inversion of the Poisson structure (6). This, however, is obstructed by the fact that the above Poisson brackets are degenerate; that is, the above algebra has Casimirs. Indeed, for any function of a single variable $f(x)$, the integral

$$
C[f]=\int \frac{d \phi}{\sqrt{\theta}} f(\rho)
$$

has vanishing Poisson brackets with $\rho$ and constitutes a Casimir. There are, thus, an infinite number of Casimirs. They are spanned, e.g., by $C_{n} \equiv C\left[x^{n+1}\right]$ for $n=$ $0,1,2, \ldots$ 
A lagrangian realization, then, of the above structure could proceed in two possible ways: we could either neutralize the Casimirs by fixing them to some numerical values, thus reducing the phase space, or append canonical partners to the Casimirs to render the Poisson brackets non-degenerate, thus augmenting the phase space. The first option, for instance, could be implemented by considering only density fields $\rho$ that are generated by canonical transformations on the single-particle phase space of some reference density function $\rho_{0}$. This is the approach that would most closely parallel the treatment in quantum mechanics.

In the next section we shall present an alternative way to realize the above structure that helps decouple the Casimirs, by transforming to a new set of dynamical variables. The transformation is motivated by the droplet picture and its significance will manifest when we apply it to the canonical formulation of droplets.

\subsection{The cartographic transformation}

To derive the desired transformation it is conceptually and analytically simpler to start from a more general framework, and later specialize to the problem at hand.

Consider a (new) classical phase space of dimension $D+1$ (which could be either even or odd). For later convenience, we single out in notation two of the coordinates of this space, $x$ and $y$, while we call the remaining coordinates $\sigma^{i}, i=1,2, \ldots D-1$. The canonical structure of the manifold is $\theta^{\mu \nu}(x, y, \sigma)$. (In the sequel we use middle greek letters for the full set of indices $(x, y, 1,2, \ldots D-1)$ and latin letters for the indices of $\sigma$, while early greek letters will be reserved for indices taking values in a $D$-dimensional space.)

The dynamical variable will be a function of one less variable, which we take to be $x: X(y, \sigma)$. The field $X$ will possess Poisson brackets to be defined shortly.

We can perform a transformation of the field $X$ into a new field $Y$ by trading one of the remaining independent variables, take it to be $y$, with $X$ itself. Specifically, we define the field $Y(x, \sigma)$ such that

$$
X(Y(x, \sigma), \sigma)=x, \quad Y(X(y, \sigma), \sigma)=y
$$

To give an explicit expression for this transformation, define the step function $\vartheta(x)$ as

$$
\vartheta(x)=\frac{1}{2}[(1+\operatorname{sgn}(x)]=1 \text { if } x>0, \quad=0 \text { else }
$$

Assuming proper monotonicity properties for $X$ we have

$$
\vartheta(X-x)=\vartheta(Y-y)
$$


[while, in general, $f(X-x) \neq f(Y-y)$ ]. Using the above and the fact that $\vartheta^{\prime}(x)=\delta(x)$, the transformation becomes

$$
Y(x, \sigma)=\int d y y \delta(Y-y)=\int d y \vartheta(Y-y)=\int d y \vartheta(X-x)
$$

while the inverse transformation is

$$
X(y, \sigma)=\int d x \vartheta(Y-y)
$$

This transformation is obviously symmetric.

It is useful to visualize the fundamental dynamical variable as a $D$-dimensional membrane (a " $D$-brane") embedded in the $D+1$-dimensional phase space. $X$ and $Y$, then, are two different ways of parametrizing this membrane in terms of its $x$ or $y$ coordinate, respectively. The transition from $X$ to $Y$ will be called a cartographic transformation. When we specialize to the case of interest, namely of a phase space density $\rho$ in $D$ phase space dimensions, $X$ will become the density, while $Y$ will effectively become a parametrization of the density in terms of overlapping droplets. This shall be explained in the subsequent sections.

To posit the fundamental Poisson brackets of the problem, define the quantity $S[A]$ for an arbitrary function $A(x, y, \sigma)$ :

$$
S[A]=\int \frac{d x d y d \sigma}{\sqrt{\theta}} A(x, y, \sigma) \vartheta(X-x)=\int \frac{d x d y d \sigma}{\sqrt{\theta}} A(x, y, \sigma) \vartheta(Y-y)
$$

We postulate the Poisson brackets of the above dynamical variables as

$$
\{S[A], S[B]\}=\int \frac{d x d y d \sigma}{\sqrt{\theta}}\{A, B\}_{s p} \vartheta(X-x)=\int \frac{d x d y d \sigma}{\sqrt{\theta}} \theta^{\mu \nu} \partial_{\mu} A \partial_{\nu} B \vartheta(X-x)
$$

where $\{A, B\}_{s p}$ are the single-particle Poisson brackets in the full phase space. The Poisson brackets (18) satisfy the Jacobi identity, as a corollary of the Jacobi identity for $\theta^{\mu \nu}$. By using (14) we can obtain equivalent expressions for the $S[A]$ and their Poison brackets in terms of $Y(x, \sigma)$.

The above Poisson brackets imply underlying Poisson brackets for the fundamental dynamical fields $X$ or $Y$. To determine them for $X$, substitute the following specific functions in the definition of $S[A]$ and $S[B]$

$$
A=\sqrt{\theta} \delta\left(y-y_{1}\right) \delta\left(\sigma-\sigma_{1}\right), \quad B=\sqrt{\theta} \delta\left(y-y_{2}\right) \delta\left(\sigma-\sigma_{2}\right)
$$

which gives $S[A]=X\left(y_{1}, \sigma_{1}\right), S[B]=X\left(y_{2}, \sigma_{2}\right)$. Plugging the above functions in (18) we obtain after some algebra

$$
\left\{X\left(y_{1}, \sigma_{1}\right), X\left(y_{2}, \sigma_{2}\right)\right\}=\sqrt{\theta_{+}(X)}\left[\theta_{+}^{x \alpha}(X)+\theta_{+}^{\alpha \beta}(X)\left(\partial_{\beta} X\right)_{+}\right] \partial_{\alpha} \delta\left(y_{-}, \sigma_{-}\right)
$$


where the indices $\alpha, \beta$ take the values $y, i$. In the above, we defined

$$
\theta_{+}^{\mu \nu}(X)=\theta^{\mu \nu}\left(X\left(y_{+}, \sigma_{+}\right), y_{+}, \sigma_{+}\right), \quad\left(\partial_{i} X\right)_{+}=\left(\partial_{i} X\right)\left(y_{+}, \sigma_{+}\right)
$$

Conversely, assuming the Poisson brackets (201) for $X$ we can show that (18) are satisfied. (This step is necessary, since the set of functions (19) used to derive (20) do not span the full space of functions of three variables.) The derivation is given below.

To alleviate the notation, for any function $f(x, y, \sigma)$ (such as $A$ or $\theta^{\mu \nu}$ ) we will drop the arguments $x, y$ and $\sigma$ we will write

$$
f(X) \equiv f(X(y, \sigma), y, \sigma),\left.\quad\left(\partial_{\alpha} f\right)(X) \equiv \partial_{\alpha} f(x, y, \sigma)\right|_{x=X}
$$

while $\partial_{\alpha} f(X)$ will stand for the total derivative of $f(X)$, both explicit and implicit through $X(y, \sigma)$. (As before, early greek indices stand for $y$ or $i$.)

The Poisson brackets of two functionals $F$ and $G$ of $X$, will be given in terms of their variations with respect to $X$ and the Poisson brackets of $X$ (20). Writing

$$
\frac{\delta F}{\delta X}=\frac{1}{\sqrt{\theta(X)}} f, \quad \frac{\delta G}{\delta X}=\frac{1}{\sqrt{\theta(X)}} g
$$

and using (20) we have

$$
\begin{aligned}
\{F, G\} & =\int d y_{1} d \sigma_{1} d y_{2} d \sigma_{2} \frac{\delta F}{\delta X_{1}} \frac{\delta G}{\delta X_{2}}\left\{X_{1}, X_{2}\right\} \\
& =\int \frac{d y d \sigma}{\sqrt{\theta(X)}} f\left[\theta^{x \alpha}(X)+\theta^{\alpha \beta}(X) \partial_{\beta} X\right] \partial_{\alpha} g
\end{aligned}
$$

We shall apply this formula for the functionals $S[A]$ in (17), whose variation is

$$
\delta S[A]=\int \frac{d x d y d \sigma}{\sqrt{\theta}} A \delta(X-x) \delta X=\int \frac{d y d \sigma}{\sqrt{\theta(X)}} A(X) \delta X
$$

We obtain

$$
\{S[A], S[B]\}=\int \frac{d y d \sigma}{\sqrt{\theta(X)}} A(X)\left[\theta^{x \alpha}(X)+\theta^{\alpha \beta}(X) \partial_{\beta} X\right] \partial_{\alpha} B(X)
$$

Expanding the total derivative $\partial_{\alpha} B(X)=\left(\partial_{\alpha} B\right)(X)+\left(\partial_{x} B\right)(X) \partial_{\alpha} X$ we have

$$
\begin{aligned}
\{S[A], S[B]\}=\int \frac{d y d \sigma}{\sqrt{\theta(X)} A(X)} & {\left[\theta^{x \alpha}(X)\left(\partial_{\alpha} B\right)(X)+\theta^{x \alpha}(X)\left(\partial_{x} B\right)(X) \partial_{\alpha} X\right.} \\
+ & \left.\theta^{\alpha \beta}(X)\left(\partial_{\alpha} B\right)(X) \partial_{\beta} X\right]
\end{aligned}
$$


By inserting $\int d x \delta(X-x)$ in the above integral we can eliminate the dependence of all quantities on $X$ and reintroduce $x$. Further, by using

$$
\delta(X-x)=-\partial_{x} \vartheta(X-x), \quad \partial_{\alpha} X \delta(X-x)=\partial_{\alpha} \vartheta(X-x)
$$

the above expression becomes

$$
\begin{aligned}
\{S[A], S[B]\}=\int \frac{d x d y d \sigma}{\sqrt{\theta}} A+ & {\left[\theta^{\alpha x} \partial_{\alpha} B \partial_{x} \vartheta(X-x)+\theta^{x \alpha} \partial_{x} B \partial_{\alpha} \vartheta(X-x)\right.} \\
+ & \left.\theta^{\alpha \beta} \partial_{\alpha} B \partial_{\beta} \vartheta(X-x)\right]
\end{aligned}
$$

We see that the terms in the bracket constitute the full expansion of the singleparticle bracket $\{B, \vartheta(X-x)\}_{s p}$. With an extra integration by parts, and using $\partial_{\mu}\left(\theta^{\mu \nu} / \sqrt{\theta}\right)=0$, we obtain the Poisson bracket (18) as desired.

A similar analysis would yield the Poisson brackets for $Y$. The result is

$$
\left\{Y\left(x_{1}, \sigma_{1}\right), Y\left(x_{2}, \sigma_{2}\right)\right\}=\sqrt{\theta_{+}(Y)}\left[\theta_{+}^{y \alpha}(Y)+\theta_{+}^{\alpha \beta}(Y)\left(\partial_{\beta} Y\right)_{+}\right] \partial_{\alpha} \delta\left(x_{-}, \sigma_{-}\right)
$$

in an obvious notation, where now $\alpha, \beta=x, i$.

To recapitulate, we have demonstrated that if the variable $X(y, \sigma)$ has Poisson brackets (20), then the transformation (15) generates a new variable $Y(x, \sigma)$ that has Poisson brackets (30).

If the canonical structure $\theta^{\mu \nu}$ is non-degenerate, the Poisson structure of $X$ or $Y$ will also be non-degenerate, the only Casimir being the trivial one corresponding to $A=1$ :

$$
C_{0}=\int \frac{d x d y d \sigma}{\sqrt{\theta}} \vartheta(X-x)=\int \frac{d x d y d \sigma}{\sqrt{\theta}} \vartheta(Y-y)
$$

The rest of the Casimirs $C_{n}$ constructed in analogy to the ones for $\rho$ are not present here (or, rather, they are all equal to $C_{0}$ ), since $\vartheta(x)^{n+1}=\vartheta(x)$ for $n=0,1,2, \ldots$ Note, further, that $S[A]$ span all functionals of $X$ or $Y$ of the form $\int f(X, y, \sigma)$ or $\int f(x, Y, \sigma)$. Indeed, any function $f(X)$ can be expanded in terms of step functions as

$$
f(X)=\int d x f^{\prime}(x) \vartheta(X-x)
$$

and with functions $A(x, y, \sigma)$ this can be done pointwise on the space $(y, \sigma)$. Therefore, the algebra of all these functionals is non-degenerate and contains no Casimirs except $C_{0}$. This non-degeneracy in the Poisson brackets of $X$ or $Y$ manifests in the appearance of the affine term proportional to $\theta^{x \alpha}$ or $\theta^{y \alpha}$. If, on the other hand, the canonical structure $\theta^{\mu \nu}$ is degenerate, this degeneracy will also be inherited by the Poisson structures of $X$ or $Y$. 


\subsection{Canonical decomposition of the density}

We are now ready to apply this formalism to the case of the density field $\rho$ on our original phase space. For this, choose the $D+1$-dimensional phase space to be our original $D$-dimensional phase space plus one additional trivial coordinate $x$. That is, choose $\theta^{\mu \nu}(y, \sigma)$ independent of $x$ given by

$$
y=\phi^{0}, \quad \sigma^{i}=\phi^{i} ; \quad \theta^{x \alpha}=0, \quad \theta^{\alpha \beta}(y, \sigma)=\theta^{\alpha \beta}\left(\phi^{0}, \phi^{i}\right)
$$

The function $X(y, \sigma)$ will be identified with the phase space density $\rho$. With the above choice it is clear that the affine term in (20) as well as all dependence on $X=\rho$ drop and we recover the standard Poisson brackets of $\rho$ (6).

This canonical structure is certainly degenerate, due to the degeneracy of the coordinate $x$, making all functions of $x$ Casimirs of the single-particle Poisson brackets. As a consequence, the Poisson structure of $X=\rho$ is also degenerate. Indeed, the set of $S[f(x)]$ for any function of $x$ alone constitute Casimirs, spanned by $S\left[x^{n}\right]$ for $n=0,1, \ldots$ Substituting $A=x^{n}$ in (17) and using the fact that $\theta$ is $x$-independent and $X=\rho$ we obtain

$$
S\left[x^{n}\right]=\frac{1}{n+1} \int \frac{d \phi}{\sqrt{\theta}} \rho^{n+1}
$$

recovering the tower of Casimirs $C_{n}$ identified before.

Perform, now, the cartographic transformation from $x$ to $y=\phi^{0}$, The hamiltonian and Poisson structure for $Y$ become

$$
H=\int \frac{d x d \phi}{\sqrt{\theta}} V(\phi) \vartheta\left(Y-\phi^{0}\right)
$$

and

$$
\left\{Y\left(x_{1}, \sigma_{1}\right), Y\left(x_{2}, \sigma_{2}\right)\right\}=\sqrt{\theta_{+}(Y)}\left[\theta_{+}^{0 \alpha}(Y)+\theta_{+}^{\alpha \beta}(Y)\left(\partial_{\beta} Y\right)_{+}\right] \partial_{\alpha} \delta\left(x_{-}, \sigma_{-}\right)
$$

Note that the above Poisson brackets do not involve any $x$-derivatives. As a result, the Poisson algebra decomposes into an infinite direct sum of mutually commuting algebras, one for each value of $x$. Each of these algebras is non-degenerate (note that (36) still contains an affine term), the only Casimir being the zero-mode of $Y$ :

$$
C(x)=\int \frac{d \phi}{\sqrt{\theta}} \vartheta\left(Y-\phi^{0}\right)
$$

The above reproduces the full set of Casimirs of the original Poisson algebra. Indeed, using $\vartheta\left(Y-\phi^{0}\right)=\vartheta(\rho-x)$ and the $x$-independence of $\theta$ we have

$$
\int d x x^{n} C(x)=\int \frac{d x d \phi}{\sqrt{\theta}} x^{n} \vartheta(\rho-x)=\frac{1}{n+1} \int \frac{d \phi}{\sqrt{\theta}} \rho^{n+1}=\frac{1}{n+1} C_{n}
$$


We therefore achieved to bring the density Poisson algebra in a decoupled form that makes its lagrangian realization possible: For fixed $x$, the algebra of $Y(x)$ can be realized in terms of an action, the only obstruction to take care being the zeromode $C(x)$. This can be done either by omitting the zero mode altogether (fix the Casimir $C(x))$ or by endowing it with a canonical partner $\Theta(x)$. Specifically, if we add an extra field $\Theta(x)$ (a function of $x$ only) with Poisson brackets

$$
\left\{\Theta\left(x_{1}\right), Y\left(x_{2}, \sigma\right)\right\}=\sqrt{\theta(Y)} \delta\left(x_{-}\right)
$$

then the full algebra becomes non-degenerate.

\subsection{Comparison to fluid dynamics}

It is useful to note that the above cartographic transformation is similar to the hodographic transformation that produces a fluid mechanical system out of a NambuGoto $D$-brane theory [22, 23]. (For a comprehensive review of this and related issues see [24.) The differences are that in our case we work in phase space while in the $D$-brane case there are independent momenta, and that in our case we only trade one variable while in the $D$-brane case $D$ spatial world-volume variables are traded for corresponding target-space variables (hodographic, which means 'path-tracing' in Greek, alludes to this point-wise mapping in time from target space to world volume space).

In the $D$-brane case this mapping has the effect of reverting from a 'lagrangian' (body-fixed) description of the fluid to an 'eulerian' one in terms of density and velocity fields. The volume-preserving reparametrization invariance of the former description (a 'particle re-labeling' transformation) is eliminated by working in terms of the reparametrization-invariant quantities of density and velocity.

In our case, something similar takes place: an underlying particle realization of the phase space density $\rho$ would introduce particle-relabeling arbitrariness, which is related to the existence of the Casimirs $C_{n}$. The cartographic transformation is more like a transition from an eulerian description in terms of a phase-space density $\rho$ (there are no velocities) to a partially lagrangian description in terms of $Y$. That $Y$ is akin to a lagrangian body-fixed variable is evident from its equation of motion as will be derived in the next section, which contains a term relevant to a particle at position $\phi^{0}=Y$. This description, however, neutralizes the Casimirs by eliminating the relabeling arbitrariness of particles along the contours $\rho=$ constant. It amounts to parametrizing the landcape of the field $\rho$ in terms of its curves of equal altitude, thus the name 'cartographic'. 
We conclude with the comment that the above transformation resolves in principle the problem of the lagrangian realization of the $\rho$-algebra, although in practice the explicit realization of the algebra of $Y(x)$ will depend on the exact form of $\theta^{\alpha \beta}(\phi)$ and may be complicated.

\section{Phase space droplet dynamics}

\subsection{Description and equation of motion}

We now turn our attention to the situation where the particles underlying the density $\rho$ are (spinless) fermions. A dense collection of fermions in the phase space will form a Fermi liquid. Semiclassically, the fermions will fill densely a region of the phase space, with one particle per volume $h^{D / 2}$. Such a state constitutes a constant density droplet of arbitrary shape, uniquely determined by its boundary. Under time evolution, the density remains constant inside the droplet (by Liouville's theorem) while each point of the boundary moves according to the single-particle equation of motion, thus deforming the shape of the droplet.

To describe the droplet it suffices to determine the shape of its $D$-1-dimensional boundary. This can be done by expressing one of the phase space coordinates on the boundary, say $\phi^{0} \equiv R$, as a function of the remaining phase space coordinates $\sigma^{i}$. So the dynamical variable is the function $R(\sigma, t)$. (For a finite droplet, it is convenient to assume that the origin of coordinates is inside the droplet and to think of $R$ as a 'radial' coordinate and $\sigma^{i}$ as 'angular' coordinates.)

The evolution of $R$ is due to two factors: first, the coordinate $R$ itself evolves according to the single-particle equation of motion; and second, the variables $\sigma^{i}$ evolve as well. A point on the boundary evolves as

$$
(R, \sigma) \rightarrow\left(R+\left.\dot{\phi}^{0}\right|_{\phi^{0}=R} d t, \sigma+\dot{\sigma} d t\right)
$$

The canonical evolution of $\phi^{0}$ at the boundary, then, gives the co-moving time derivative of the function $R(\sigma)$ :

$$
\dot{R}+\dot{\sigma}^{i} \partial_{i} R=\theta^{0 i}(R)\left(\partial_{i} V\right)(R)
$$

Expressing $\dot{\sigma}^{i}$ in terms of its equation of motion gives the time derivative of $R$ :

$$
\dot{R}=\theta^{0 i}(R)\left(\partial_{i} V\right)(R)-\theta^{i 0}(R)\left(\partial_{0} V\right)(R) \partial_{i} R-\theta^{i j}(R)\left(\partial_{j} V\right)(R) \partial_{i} R
$$


The total $\sigma$-derivative of the hamiltonian function on the boundary $V(R)$ is

$$
\partial_{i} V(R)=\left(\partial_{i} V\right)(R)+\left(\partial_{0} V\right)(R) \partial_{i} R
$$

Using the above, as well as the antisymmetry of $\theta^{i j}$, we can express the equation of motion for $R$ in terms of the total derivative of $V(R)$ as:

$$
\dot{R}=\theta^{0 j}(R) \partial_{j} V(R)-\theta^{i j}(R) \partial_{j} V(R) \partial_{i} R
$$

This is the basic equation of motion for the droplet. It is first-order in time and phase space derivatives and thus fundamentally chiral.

It is worth noting that, if the coordinate $\phi^{0}$ is chosen to parametrize the potential (that is, surfaces $\phi^{0}=$ constant are equipotential), $V=V\left(\phi^{0}\right)$, the second term above drops and we get

$$
\dot{R}=\theta^{0 i}(R) \partial_{i} V(R)
$$

In the special case when $\theta^{0 i}(R)$ is nonzero only for a single value of the index $i$ (there is a global variable conjugate to $\phi^{0}$, call it $\phi^{1}$ ), and is a function only of $\phi^{0}$, the above equation becomes

$$
\dot{R}=\theta^{01}(R) V^{\prime}(R) \partial_{1} R
$$

which can easily be solved by a hodographic transformation, interchanging $R$ and $\phi^{1}$. Such choices will be useful later, but for now we remain fully general.

We should warn that depending on its topology the droplet may have more than one boundaries. In such cases we would need to introduce several commuting boundary fields $R_{n}$, one for each boundary. Similarly, the boundary could intersect $\sigma=$ constant lines at more than one $\phi^{0}$, in which case we would again need to introduce several boundary fields, one for each branch, with appropriate matching conditions tying them into a unique boundary.

\subsection{Canonical realization of droplet dynamics}

The above dynamics arise from a hamiltonian reduction of the full density canonical structure: a constant-density droplet of arbitrary shape constitutes a particular class of density functions and thus a submanifold of the full manifold of configurations for $\rho$, of the form

$$
\rho=\rho_{o} \vartheta\left(R-\phi^{0}\right)
$$


We need to project the canonical two-form of $\rho$ on this submanifold. This can be easiest done in the cartographic transformed variable. Applying (15) for this $\rho$, and noting that

$$
\vartheta\left(\rho_{o} \vartheta\left(R-\phi^{0}\right)-x\right)=\vartheta\left(R-\phi^{0}\right) \vartheta\left(\rho_{o}-x\right)
$$

we obtain

$$
Y(x, \sigma)=\vartheta\left(\rho_{o}-x\right) R(\sigma)
$$

So the reduction consists in putting all $Y(x)=0$ for $x>\rho_{o}$ and all $Y(x)$ equal to each other for $x<\rho_{o}$. This second class constraint is easily implemented, giving a canonical two-form for $R$

$$
\Omega_{R}=\int d x \vartheta\left(\rho_{o}-x\right) \Omega_{Y(x)}=\rho_{o} \Omega_{Y}
$$

where we used the decoupling in $x$ and $x$-independence of the canonical structure of $Y(x)$. Therefore, the Poisson brackets for $R$ are the same as those of $Y(x)$ times $1 / \rho_{o}$ :

$$
\left\{R\left(\sigma_{1}\right), R\left(\sigma_{2}\right)\right\}=\frac{\sqrt{\theta_{+}(R)}}{\rho_{o}}\left[\theta_{+}^{0 i}(R)+\theta_{+}^{i j}\left(\partial_{j} R\right)_{+}\right] \partial_{i} \delta\left(\sigma_{-}\right)
$$

The corresponding hamiltonian is

$$
H=\int \frac{d x d \phi}{\sqrt{\theta}} V(\phi) \vartheta\left(Y-\phi^{0}\right)=\rho_{o} \int \frac{d \phi}{\sqrt{\theta}} V(\phi) \vartheta\left(R-\phi^{0}\right)
$$

The above Poisson structure and hamiltonian encode the full dynamics of the droplet. We can explicitly verify that they reproduce the equation of motion (44) as the canonical evolution

$$
\dot{R}=\{R, H\}
$$

Note that the above hamiltonian is defined in the bulk of the droplet, although the equation of motion refers only to the boundary.

The constant $\rho_{o}$ is irrelevant for classical dynamics. The semiclassical interpretation of the droplet, however, fixes the value $\rho_{o}=1 /(2 \pi \hbar)^{D / 2}$, which will be important for quantization.

We conclude this section with the following remarks:

1. The droplet situation closely parallels the one discussed in section 3 , in dimension $D$ rather than $D+1$ : the boundary of the droplet plays the role of the $D-1$-brane in the phase space parametrized by its $\phi^{0}$-coordinate $R$. The hamiltonian is $H=\rho_{o} S[V]$ and the Poisson brackets (18) are the statement that the droplet density satisfies the proper Poisson density algebra (6). 
2. The Poisson brackets (51) of $R$ contain an affine 'chiral' part as well as an ordinary Poisson density structure (the second term in the bracket). over the gauge manifold $\left\{\sigma^{i}\right\} / \phi_{0}$. The quotient arises because $\theta^{i j}$ is degenerate, being odddimensional, and effectively the variable conjugate to $\phi^{0}$ drops out.

3. (51) satisfies the Jacobi identity, as a corollary of the Jacobi identity of the full Poisson brackets for $Y$, although its direct check is highly nontrivial. In the special case when $\theta^{\alpha \beta}$ is independent of $\phi^{0}$ the affine and linear terms decouple and individually satisfy the Jacobi identity. In the generic case, however, both terms are needed to satisfy the identity.

4. The Casimirs of the original density for the droplet become $C[x]=\vartheta\left(\rho_{o}-x\right) C_{0}$ or $C_{n}=C_{0}$. So they are all neutralized, the only essential Casimir being the total particle number $C_{0}=N$.

\subsection{The linearized action}

Assuming that the droplet deviates only slightly from an equilibrium configuration, we can analyze its motion as a perturbation of its equilibrium shape. This will be useful for large droplets (large number of particles) whose motion involves only boundary perturbations.

Consider an equilibrium configuration in which the droplet fills the phase space up to an energy level $V_{o}$. That is, the boundary function $R_{o}(\sigma)$ is such that

$$
V\left(R_{o}(\sigma), \sigma\right)=V_{o}=\text { constant }
$$

Such a function obviously satisfies the equation of motion for $R$ with $\dot{R}=0$. A perturbation of the droplet around this configuration can be written as $R=R_{o}+\chi$, $\chi \ll R_{o}$. Correspondingly, the energy function is perturbed as

$$
V(\sigma)=V_{o}+\omega_{o}(\sigma) \chi(\sigma), \quad \text { where } \omega_{o}=\left(\partial_{0} V\right)\left(R_{o}\right)
$$

So to lowest order in $\chi$ the equation of motion (44) becomes:

$$
\dot{\chi}=\left(\theta_{o}^{0 i}-\theta_{o}^{j i} \partial_{j} R_{o}\right) \partial_{i}\left(\omega_{o} \chi\right)
$$

where $\theta_{o}^{\alpha \beta}=\theta^{\alpha \beta}\left(R_{o}(\sigma), \sigma\right)$.

Define the differential operator

$$
\mathcal{L}=u^{i} \partial_{i}=\left(\theta_{o}^{0 i}-\theta_{o}^{j i} \partial_{j} R_{o}\right) \partial_{i}
$$


Then the above equation becomes simply

$$
\dot{\chi}=\mathcal{L}\left(\omega_{o} \chi\right)
$$

Similarly, to lowest order in $\chi$ we have:

$$
\left\{\chi\left(\sigma_{1}\right), \chi\left(\sigma_{2}\right)\right\}=\frac{\sqrt{\theta_{o}\left(\sigma_{+}\right)}}{\rho_{o}} \mathcal{L}\left(\sigma_{+}\right) \delta\left(\sigma_{1}-\sigma_{2}\right)
$$

and

$$
H=H_{o}+\rho_{o} V_{o} \int \frac{d \sigma}{\sqrt{\theta_{o}}} \chi+\int \frac{d \sigma}{\sqrt{\theta_{o}}} \frac{1}{2} \omega_{o} \chi^{2}
$$

$H_{o}$ is a constant and can be dropped. The next term, linear in $\chi$, is simply $\rho_{o} V_{o} C_{0}$ plus a constant; it is therefore a Casimir and does not contribute to the equations of motion. It can be set to zero as an initial condition, corresponding to a constantvolume perturbation of the droplet (total number of particles constant), and we end up with a quadratic hamiltonian.

We can derive the above linearized structure from a lagrangian and make contact with the chiral action of Karabali and Nair. To achieve this, consider that the deformation of the droplet is generated by an infinitesimal canonical transformation on the phase space $\varphi(\sigma)$ :

$$
\left(R_{o}, \sigma\right) \rightarrow\left(R_{o}+\theta_{o}^{0 i} \partial_{i} \varphi, \sigma+\theta_{o}^{i j} \partial_{j} \varphi\right)
$$

or

$$
\chi=\left(\theta_{o}^{0 i}-\theta_{o}^{j i} \partial_{j} R_{o}\right) \partial_{i} \varphi=\mathcal{L} \varphi
$$

With this redefinition, the Poisson brackets of $\varphi$ will involve the inverse of the operator $\mathcal{L}$ and so its canonical two-form will involve $\mathcal{L}$ itself. We obtain the action

$$
S=\int d t \frac{d \sigma}{\sqrt{\theta_{o}}} \frac{1}{2}\left[\dot{\varphi} \mathcal{L} \varphi-\omega_{o}(\mathcal{L} \varphi)^{2}\right]
$$

Note that $\mathcal{L}$ is an anti-self-adjoin operator on $\sigma$, due to the relation

$$
\partial_{i}\left(\frac{u^{i}}{\sqrt{\theta_{o}}}\right)=0
$$

which is a corollary of (8). The equation of motion from this action becomes

$$
\mathcal{L} \dot{\varphi}=\mathcal{L}\left(\omega_{0} \mathcal{L} \varphi\right)
$$

which, upon putting $\chi=\mathcal{L} \varphi$, reproduces (58). 
To make contact with the action of Karabali and Nair, note that in [17] they effectively made the choice $V=V(R)$ (their coordinate is 'normal' to the boundary) and thus $R_{o}=$ constant. In this case $\mathcal{L}=\theta_{o}^{0 i} \partial_{i}$. They also made the choice $\omega_{o}=$ constant. With these choices, our action (63) becomes identical to theirs.

The zero modes of $\mathcal{L}, \mathcal{L} \varphi=0$, do not generate any transformation for $R$ and should, thus, be discarded. The equation of motion for $\varphi$, in this case, can be written in the first-order form:

$$
\dot{\varphi}=\omega_{0} \mathcal{L} \varphi
$$

On the other hand, there may be topological obstructions to the map from $\varphi$ to $\chi$, that is, there may be perturbations $\chi$ that cannot be generated by a (single-valued) function $\varphi$. This is, again, related to zero modes of $\mathcal{L}$ : if $\mathcal{L} \chi=0$, then obvioulsy such a $\chi$ cannot be generated by any $\varphi$.

These zero modes are, in fact, related to Casimirs of the linearized Poisson brackets (59). Defining $\mathcal{L}$-eigenfunctions

$$
\mathcal{L} \chi_{\lambda}=i \lambda \chi_{\lambda}, \quad \chi_{\lambda}^{*}=-\chi_{-\lambda}
$$

( $\lambda$ could be either discrete of continuous), the projection of $\chi$ on any of the zero modes $\chi_{0}$

$$
c_{0}=\int \frac{d \sigma}{\sqrt{\theta_{o}}} \chi_{0}^{*} \chi
$$

commutes with $\chi$ and is a Casimir of (59). The lagrangian realization $\chi=\mathcal{L} \varphi$ sets these Casimirs to zero. It should be stressed, however, that the above Casimirs are an artifact of the linearized approximation and are lifted in the full Poisson algebra, since the (omitted) linear in $\chi$ piece endows them with nontrivial Poisson brackets.

We conclude by pointing out that the operator $\omega_{0} \mathcal{L}$ generates classical particle motion on the surface $V=V_{o}$. So $\varphi$ moves simply as a scalar co-moving field on this surface. $\chi$, on the other hand, moves as a scalar density with $\omega_{o}$ as a weight factor. This is due to the fact that classical motion on the $V=V_{o}$ surface also rescales distances away from the surface, such as $\chi . \omega_{o} \chi$ is a scalar.

\section{Quantization}

The chiral theories obtained in the previous section describe a collection of fermions in terms of a bosonic field $R$ or $\chi$. This description is still semiclassical. Quantization of these theories should reproduce the full quantum mechanics of the Fermi liquid for a large number of fermions $N$. 
This is, indeed, the case for $D=2$, as is well established and we shall demonstrate below. For higher dimensions, however, the above chiral bosonic theory will in general have a continuous infinity of sectors, parametrized by the quotient manifold $\{\sigma\} / \phi^{0}$, that is, the manifold of orbits on the phase space spanned by $\sigma^{i}$ generated by $\phi^{0}$. (See remark 2. in section 3.2). Its quantization, therefore, would overcount the degrees of freedom and would not capture the correct physics of the fermion system.

\subsection{Quantization of the linearized $D=2$ action}

We shall start by studying the most tractable case, which is the linearized theory in 2 phase space dimensions. This theory would describe low-lying excitations of a Fermi liquid in one spatial dimension, or edge excitations in the two-dimensional (integer) quantum Hall state. The quantization of this theory is fairly standard and will be stated here for completeness.

The phase space consists of $\phi^{0}$ and a unique other variable, call it $\sigma$. For concreteness, we shall assume that $\sigma$ is periodic with period $L$. In this case $\mathcal{L}=\sqrt{\theta_{o}} \partial_{\sigma}$ and $\rho_{o}=1 /(2 \pi \hbar)$. The hamiltonian and Poisson brackets become

$$
\left\{\chi\left(\sigma_{1}\right), \chi\left(\sigma_{2}\right)\right\}=2 \pi \hbar \sqrt{\theta_{o}\left(\sigma_{+}\right)} \delta^{\prime}\left(\sigma_{1}-\sigma_{2}\right)
$$

and

$$
H=\frac{1}{2 \pi \hbar} \int_{0}^{L} \frac{d \sigma}{\sqrt{\theta_{o}}} \frac{1}{2} \omega_{o} \chi^{2}
$$

We may change variable from $\sigma$ to a new variable $\tau$ defined by $d \sigma=\omega_{o} \sqrt{\theta_{o}} d \tau$. This variable satisfies $\omega_{o} \mathcal{L} \tau=1$; since $\omega_{o} \mathcal{L}$ generates classical particle motion on $R_{o}, \tau$ is the time of flight of a particle moving on the equipotential line $\phi^{o}=R_{o}$. It is also periodic with a period

$$
T=\int_{0}^{L} \frac{d \sigma}{\omega_{o} \sqrt{\theta_{o}}}
$$

We also define a rescaled variable $\alpha=\rho_{o} \omega_{o} \chi=\omega_{o} \chi / 2 \pi \hbar$. In terms of $\alpha(\tau)$ the hamiltonian and Poisson brackets become

$$
\left\{\alpha\left(\tau_{1}\right), \alpha\left(\tau_{2}\right)\right\}=\frac{1}{2 \pi \hbar} \delta^{\prime}\left(\tau_{1}-\tau_{2}\right), \quad H=2 \pi \hbar \int_{0}^{T} d \tau \frac{1}{2} \alpha^{2}
$$

The above is an abelian chiral algebra and a quadratic hamiltonian. Quantization can proceed now in a standard way. The quantum commutator of $\alpha$ is $i \hbar$ times the classical Poisson brackets. In terms of the Fourier modes

$$
\alpha_{n}=\int_{0}^{T} d \tau \alpha(\tau) e^{-i n \frac{2 \pi \tau}{T}}
$$


we obtain the normal-ordered hamiltonian and algebra

$$
\left[\alpha_{n}, \alpha_{m}\right]=n \delta_{n+m}, \quad H=\frac{2 \pi \hbar}{T} \sum_{n>0} \alpha_{-n} \alpha_{n}+\frac{\pi \hbar}{T} \alpha_{o}^{2}
$$

The Casimir $\alpha_{0}$ represents variations of the total particle number $N$ and will be set to zero, since we work at fixed $N$. Each pair $\left(\alpha_{-n}, \alpha_{n}\right)$ is a quantum oscillator with quanta of size $n$, contributing to the energy quanta of size $\hbar 2 \pi n / T$. Calling $b_{n}=0,1,2, \ldots$ the excitation number of oscillator $\alpha_{n}$, the energy eigenvalues are

$$
E=\sum_{n>0} \frac{2 \pi \hbar}{T} n b_{n}
$$

We may compare this to the states of the fermionic theory. The ground state consists of a Fermi sea with the first $N$ single-particle levels occupied by fermions. Low-lying states involve excitations of particles near the Fermi level, that is, near the $N$-th single-particle energy eigenvalue.

The highly excited energy levels of a particle with hamiltonian $V$ in the above phase space will be given with good accuracy by the WKB approximation. The spacing between these levels becomes $\hbar$ times the frequency of classical periodic motion at this energy, that is, $\hbar 2 \pi / T$. At large $N$ this becomes exact. So the single-particle spectrum becomes equidistant with the above spacing.

The excitations around the Fermi sea can be parametrized in terms of the discrete jumps of single fermions to higher single-particle states. Enumerating fermions in terms of their position in the ground state with respect to the Fermi level ( 1 at the top, 2 the one below etc), their excitations will be $f_{n}=0,1,2, \ldots$ and the total excitation energy becomes

$$
E_{f}=\sum_{n} \frac{2 \pi \hbar}{T} f_{n}
$$

In order to avoid overcounting and respect Pauli exclusion, we must not allow any particle to jump higher than the particle above it; that is

$$
f_{n+1} \leq f_{n}, \quad n=1,2, \ldots
$$

We can now establish a mapping between the bosonic and fermionic states, by parametrizing the $f_{n}$ as

$$
f_{n}=\sum_{k \geq n} b_{n}, \quad b_{n}=f_{n}-f_{n+1}, \quad n=1,2, \ldots
$$

This gives a one-to-one correspondence between the unrestricted integers $b_{n}$ and the restricted integers $f_{n}$ satisfying (177). The energy of the fermionic system in terms 
of this parametrization becomes identical to the bosonic energy (75), demonstrating the full equivalence between the two systems. This is the standard bosonization picture in terms of phonon excitations of the Fermi sea.

The above analysis in the linearized chiral action shows that it describes the fermionic system to the leading order in large- $N$. Variations of the spacing of the levels for finite $N$ will induce $1 / N$ corrections. Further, large excitations depleting the Fermi sea give rise to nonperturbative effects. The linearized theory misses both corrections. As we will demonstrate in the next section, the full nonlinear theory captures $1 / N$ corrections but still misses nonperturbative effects.

\subsection{Quantization of nonlinear theories in $D=2$}

The quantization of general nonlinear theories even in $D=2$ is nontrivial. To make the task tractable, we shall assume that we have identified Darboux coordinates. So $\theta^{01}=1$, the only dynamical input being the energy function $V\left(\phi^{0}, \sigma\right)$. With an additional canonical transformation we can make the potential to depend only on the coordinate $\phi^{0}$. The coordinate $\phi^{1}=\sigma$ will be assumed periodic, reflecting the fact that $V=$ constant lines should be compact so that the system have discrete levels. Finally, with a residual coordinate transformation we may make the periodicity of $\sigma$ to be independent of $\phi^{0}$ and equal to $2 \pi$. (Although a canonical transformation could make $V=\phi^{0}$, apparently trivializing the problem, this would cause the conjugate variable $\sigma$ to have a $\phi^{0}$-dependent periodicity making the situation unwieldy.) The topology of the $\phi_{0}$ direction will be left unspecified.

We define the quantum field $\alpha=\rho_{o} R=R / 2 \pi \hbar$, which is essentially the chiral fermion density in the $\sigma$ direction. The quantum commutation relations and hamiltonian become

$$
\left[\alpha\left(\sigma_{1}\right), \alpha\left(\sigma_{2}\right)\right]=\frac{i}{2 \pi} \partial_{\sigma} \delta\left(\sigma_{1}-\sigma_{2}\right), \quad H=\frac{1}{2 \pi \hbar} \int d \sigma U(2 \pi \alpha)
$$

where $U^{\prime}=V$. $\alpha$ is again a current algebra variable. Constant and linear terms in $U$ are irrelevant, representing trivial shifts in energy and a Casimir. Further, a quadratic term in $U$ commutes with any hamiltonian of the above form, since it corresponds to the conserved momentum in the $\sigma$ direction, and can be separately diagonalized.

A quadratic $U$ is the simplest situation. This would correspond to a harmonic oscillator potential, with $\phi^{0}=\frac{1}{2} p^{2}+\frac{1}{2} x^{2}$ and $\tan \sigma=x / p$, or to a quantum Hall droplet on a cylinder with a linear potential along the cylinder axis. (This would, 
actually, have two separate boundaries.) Such a $U$ has already been treated as the linearized case of the previous section. The results, in that case, are exact to all orders in $1 / N$, since the harmonic oscillator potential has indeed equidistant levels with spacing $\hbar$ times its classical period.

To analyze a generic potential, we shall first write $\alpha=\alpha_{o}+\tilde{\alpha}$, where the Casimir $\alpha_{o}$ is the zero mode of $\alpha$ and $\tilde{\alpha}$ integrates to zero. The particle number is $N=$ $2 \pi \rho_{o} R_{o}=2 \pi \alpha_{o}$, so $\alpha_{o}$ is of order $N$, while we assume that the fluctuating part $\tilde{R}$ is of order $N^{0}$. Writing $U_{o}^{\prime}=U^{\prime}\left(R_{o}\right)$ etc., we may expand the hamiltonian as

$$
\frac{1}{2 \pi \hbar} \int d \sigma U\left(2 \pi \alpha_{o}+2 \pi \tilde{R}\right)=\frac{U_{o}}{\hbar}+2 \pi \hbar \int d \sigma\left[\frac{U_{o}^{\prime \prime}}{2} \tilde{\alpha}^{2}+2 \pi \hbar \frac{U_{o}^{\prime \prime \prime}}{6} \tilde{\alpha}^{3}+\ldots\right]
$$

Each subsequent term in the expansion is subleading in $\hbar$ and $1 / N$. The leading term is the quadratic one, which has already been treated and commutes with all subsequent terms. So we may omit it and examine only the subleading corrections to this hamiltonian. We see that the first nontrivial term, of order $1 / N$ is the cubic one.

A pure cubic $U$ would correspond to free particles on a circle, with $\phi^{0}=p$ and $\sigma=x$. The cubic hamiltonian with two chiral fields $R$ (representing the two Fermi levels) is also known as the fermionic collective field theory [3], [8]. It is well established that the cubic bosonic hamiltonian reproduces exactly the states of the fermionic system to all orders in the $1 / N$ expansion (which stops at $O(1 / N)$ in this case) 3$]$.

We conclude that the nonlinear hamiltonian for any function $U(R)$ reproduces the correct quantum mechanical results at least up to order $1 / N$. For higher orders we should also include derivative terms, which would arise from renormalization and start at order $1 / N^{2}$. Nonperturbative effects connected to the depletion of the Fermi sea will cause corrections of order $e^{-O(N)}$ and will always be missed.

\subsection{Anyons, Calogero, FQHE states}

In all previous considerations we took the density of the droplet to be $\rho_{o}=1 / 2 \pi \hbar$. Although this is sensible from the fermion point of view, corresponding to one fermion per quantum state, it is not really required. Both the classical and the quantum theory are well-defined for any $\rho_{o}$.

We may, thus, choose to work with $\rho_{o}=\nu / 2 \pi \hbar$, where $\nu$ represents the fraction of the single-particle states occupied by particles and can take any value. We will obtain a semiclassical description of particles with generalized exclusion statistics 
where each particle occupies $\ell=1 / \nu$ states. For $D=2$ such a system would describe either interacting Calogero-Sutherland particles with strength of the inverse-square two-body potential $\ell(\ell-1)$ [25], 26], or anyons in the lowest Landau level with statistical exchange phase $\alpha_{s}=\pi \ell$ [27], or Laughlin states in the fractional quantum Hall effect (FQHE) with exponent $\ell$ [28].

Quantization of this system along the lines of the previous section in terms of the density field $\alpha=\rho_{o} R=R / 2 \pi \hbar \ell$ leads to the algebra and hamiltonian

$$
\begin{gathered}
{\left[\alpha\left(\sigma_{1}\right), \alpha\left(\sigma_{2}\right)\right]=\frac{i}{2 \pi \ell} \partial_{\sigma} \delta\left(\sigma_{1}-\sigma_{2}\right)} \\
H=\frac{1}{2 \pi \hbar \ell} \int d \sigma U(2 \pi \hbar \ell \alpha)
\end{gathered}
$$

For a quadratic $U$ corresponding to a linear potential $V(R)$, which again describes particles in a harmonic oscillator potential, or a linear potential on the cylinder for the lowest Landau level, the results are identical to the free fermion case $(\ell=1)$, as can be seen by working with the rescaled field $\sqrt{\ell} \alpha$ that eliminates $\ell$ from both (81) and (82). Indeed, it is known that the energy eigenstates of the Calogero model [29], or of anyons in the lowest Landau level [30]-32], or of Laughlin particles, in a harmonic potential are identical to those of fermions up to a ground state shift. The density and particle correlation functions, on the other hand, constructed in terms of $\alpha$ or its exponential, will exhibit nontrivial scaling due to the appearance of $\ell$ in (81).

For a cubic $U$, corresponding to free particles on a periodic domain, the above will give the leading $1 / N$ terms of the collective field description of the Calogero model and will reproduce the spectrum to leading order in $1 / N$ [33], 34]. We see that the exact results obtained for fermions $(\ell=1)$ in the cubic case do not carry over to the Calogero/anyon/FQHE case. In the limit of large $N$ and fixed gap for the low-lying states (the conformal field theory limit), at any rate, the above theory reproduces the correct spectrum and correlations.

\section{Conclusions and discussion}

We have presented an analysis of the phase space dynamics of droplets in an arbitrary phase space and derived their hamiltonian and canonical structure. In the linearized approximation we recovered the chiral actions of edge excitations. The nonlinear hamiltonian classically describes arbitrary deformations of the droplet and quantum mechanically captures $1 / N$ corrections, at least in the two dimensional case. 
The transition from the hamiltonian to an action in the full nonlinear case is still an open issue, due to the nontrivial form of the Poisson brackets (51). If we work with Darboux coordinates, and choose one of them for $R$, the algebra becomes affine. The advantage of the linearized case is that the right hand side of (151) becomes a constant and we have essentially a generalized Heisenberg algebra that can be easily realized with a lagrangian.

The theories obtained here are first-order and chiral, in the same sense that propagation on a phase space is chiral since from each point it proceeds to a unique direction. They represent a phase space bosonization of the fermionic systems they describe. This bosonization, however, fails quantum mechanically. First, there is the possibility that the quantum action will get renormalized. This is not fatal and we have already commented on it in the last section. Secondly, and more importantly, in dimensions higher than two the linearized effective action describes chiral propagation along the direction of classical motion, while the remaining of the phase coordinates are inert. They act thus as parameters in the theory, giving rise to an infinity of modes which will in general overcount the quantum mechanical modes. Nontrivial topology of the flow lines on the surface of the droplet may connect and reduce some of these modes, but it is unlikely that we will get the correct description.

On a more speculative level, the fractional quantum Hall state has been formulated in terms of a noncommutative Chern-Simons field theory [35], and its boundary excitations can be described as a matrix model [36]. These descriptions capture essential quantum properties of the state, such as the discrete nature of the underlying constituent electrons and their statistical repulsion, at the semiclassical level. It would be interesting to examine if these (matrix) features can be incorporated in the phase space description of fermions or, in general, particles with mutual exclusion.

Finally, we only dealt with abelian theories. The nonabelian case requires the formulation of phase space droplets for several flavors or spin states of fermions. This and other related matters will be dealt with in a different publication.

Acknowledgements: I am thankful to Roman Jackiw, Hans Hansson, Dimitra Karabali, Parameswaran Nair and David Schmeltzer for useful comments on the manuscript. This research was supported in part by the National Science Foundation under grant PHY-0353301 and by the CUNY Research Foundation under grant PSCCUNY-66565-0035. 


\section{References}

[1] S. R. Coleman, "Quantum Sine-Gordon Equation As The Massive Thirring Model," Phys. Rev. D 11, 2088 (1975).

[2] S. Mandelstam, "Soliton Operators For The Quantized Sine-Gordon Equation," Phys. Rept. 23 (1976) 307.

[3] A. Jevicki and B. Sakita, "The Quantum Collective Field Method And Its Application To The Planar Limit," Nucl. Phys. B 165, 511 (1980);

[4] S. R. Das and A. Jevicki, "String Field Theory And Physical Interpretation Of D = 1 Strings," Mod. Phys. Lett. A 5, 1639 (1990).

[5] E. Witten, "Nonabelian Bosonization In Two Dimensions," Commun. Math. Phys. 92, 455 (1984).

[6] G. W. Semenoff, "Canonical Quantum Field Theory With Exotic Statistics," Phys. Rev. Lett. 61, 517 (1988); G. W. Semenoff and P. Sodano, "Exotic Spin And Statistics In (2+1)-Dimensional Canonical Quantum Field Theory," Nucl. Phys. B 328, 753 (1989).

[7] T. Matsuyama, "Canonical Structures And Bose-Fermi Transmutations In (2+1)-Dimensional U(1) Gauge Theories," Phys. Lett. B 228, 99 (1989).

[8] J. Polchinski, "Classical limit of (1+1)-dimensional string theory," Nucl. Phys. B 362, 125 (1991).

[9] X. G. Wen, "Chiral Luttinger Liquid And The Edge Excitations In The Fractional Quantum Hall States," Phys. Rev. B 41, 12838 (1990); D. H. Lee and X. G. Wen, Phys. Rev. Lett. 66, 1765 (1991).

[10] M. Stone, Phys. Rev. B 42 (1990) 8399; "Edge Waves In The Quantum Hall Effect," Annals Phys. 207, 38 (1991).

[11] J. Frohlich and T. Kerler, "Universality In Quantum Hall Systems," Nucl. Phys. B 354 (1991) 369.

[12] S. Iso, D. Karabali and B. Sakita, "One-dimensional fermions as twodimensional droplets via Chern-Simons theory," Nucl. Phys. B 388, 700 (1992) arXiv:hep-th/9202012; Phys. Lett. B 296, 143 (1992) arXiv:hep-th/9209003. 
[13] A. Cappelli, G. V. Dunne, C. A. Trugenberger and G. R. Zemba, "Conformal symmetry and universal properties of quantum Hall states," Nucl. Phys. B 398, 531 (1993) arXiv:hep-th/9211071.

[14] S. C. Zhang and J. p. Hu, "A Four Dimensional Generalization of the Quantum Hall Effect," Science 294, 823 (2001) arXiv:cond-mat/0110572; Phys. Rev. B 41, 12838 (1990) arXiv:cond-mat/0112432; B. A. Bernevig, C. H. Chern, J. P. Hu, N. Toumbas and S. C. Zhang, "Effective field theory description of the higher dimensional quantum Hall liquid," Annals Phys. 300, 185 (2002) arXiv:cond-mat/0206164.

[15] M. Fabinger, "Higher-dimensional quantum Hall effect in string theory," JHEP 0205, 037 (2002) arXiv:hep-th/0201016.

[16] Y. X. Chen, B. Y. Hou and B. Y. Hou, "Non-commutative geometry of 4-dimensional quantum Hall droplet," Nucl. Phys. B 638, 220 (2002) arXiv:hep-th/0203095|; Y. X. Chen, "Matrix models of 4-dimensional quantum Hall fluids," arXiv:hep-th/0209182.

[17] D. Karabali and V. P. Nair, "Quantum Hall effect in higher dimensions," Nucl. Phys. B 641, 533 (2002) arXiv:hep-th/0203264; "The effective action for edge states in higher dimensional quantum Hall systems," Nucl. Phys. B 679, 427 (2004) arXiv:hep-th/0307281; "Edge states for quantum Hall droplets in higher dimensions and a generalized WZW model," arXiv:hep-th/0403111.

[18] H. Elvang and J. Polchinski, "The quantum Hall effect on R**4," arXiv:hep-th/0209104.

[19] G. Sparling, "Twistor theory and the four-dimensional Quantum Hall effect," arXiv:cond-mat/0211679 D. Mihai, G. Sparling and P. Tillman, "Non-commutative time, the quantum Hall effect and twistor theory," arXiv:cond-mat/0401224.

[20] S. Bellucci, P. Y. Casteill and A. Nersessian, "Four-dimensional Hall mechanics as a particle on CP(3)," Phys. Lett. B 574, 121 (2003) arXiv:hep-th/0306277.

[21] B. Sakita, "Collective variables of fermions and bosonization," Phys. Lett. B 387, 118 (1996) arXiv:hep-th/9607047; R. Ray and B. Sakita, "Bulk and edge excitations of a $\nu=1$ Hall ferromagnet," arXiv:cond-mat/0105626. 
[22] M. Bordemann and J. Hoppe, "The Dynamics of relativistic membranes. 1. Reduction to two-dimensional fluid dynamics," Phys. Lett. B 317, 315 (1993) arXiv:hep-th/9307036.

[23] R. Jackiw and A. P. Polychronakos, "Dynamical Poincare symmetry realized by field-dependent diffeomorphisms," Proc. Steklov Inst. Math. 226, 193 (1999) arXiv:hep-th/9809123|; "Fluid dynamical profiles and constants of motion from d-branes," Commun. Math. Phys. 207, 107 (1999) arXiv:hep-th/9902024.

[24] R. Jackiw, V. P. Nair, S. Y. Pi and A. P. Polychronakos, "Perfect fluid theory and its extensions," arXiv:hep-ph/0407101

[25] A. P. Polychronakos, "Nonrelativistic Bosonization And Fractional Statistics," Nucl. Phys. B 324, 597 (1989); "Generalized statistics in one dimension," Les Houches Session LXIX (1998) arXiv:hep-th/9902157.

[26] S. B. Isakov, "Fractional statistics in one-dimension: Modeling by means of $1 / \mathrm{x}^{2}$ interaction and statistical mechanics," Int. J. Mod. Phys. A 9 (1994) 2563.

[27] J. M. Leinaas and J. Myrheim, "Intermediate statistics for vortices in superfluid films," Phys. Rev. B 37, 9286 (1988).

[28] R. B. Laughlin, 'The Quantum Hall Effect,' R. E. Prange and S. M. Girvin (Eds), p. 233.

[29] F. Calogero, "Solution Of The One-Dimensional N Body Problems With Quadratic And/Or Inversely Quadratic Pair Potentials," J. Math. Phys. 12 (1971) 419 .

[30] G. V. Dunne, A. Lerda and C. A. Trugenberger, "Anyons in a magnetic field: Landau levels and vertex operator representation," Int. J. Mod. Phys. B 5, 1675 (1991).

[31] A. P. Polychronakos, "Exact anyonic states for a general quadratic Hamiltonian," Phys. Lett. B 264, 362 (1991).

[32] J. Grundberg, T. .H. Hansson, A. Karlhede and E. Westerberg, "Landau levels for anyons," Phys. Rev. B 44, 8373 (1991).

[33] I. Andric and V. Bardek, "1/N Corrections In Calogero Type Models Using The Collective Field Method," J. Phys. A 21 (1988) 2847. 
[34] J. A. Minahan and A. P. Polychronakos, "Density correlation functions in Calogero-Sutherland models," Phys. Rev. B 50, 4236 (1994) arXiv:hep-th/9404192.

[35] L. Susskind, "The quantum Hall fluid and non-commutative Chern Simons theory," arXiv:hep-th/0101029.

[36] A. P. Polychronakos, "Quantum Hall states as matrix Chern-Simons theory," JHEP 0104, 011 (2001) arXiv:hep-th/0103013; "Quantum Hall states on the cylinder as unitary matrix Chern-Simons theory," JHEP 0106, 070 (2001) arXiv:hep-th/0106011. 